\title{
The transfer matrix with Kogut-Susskind fermions
}

\author{
Fabrizio Palumbo * \\ INFN - Laboratori Nazionali di Frascati \\ P. O. Box 13, I-00044 Frascati, ITALIA \\ Internet: palumbof@lnf.infn.it
}

July 27,2021

\begin{abstract}
A transfer matrix for gauge theories on a lattice with KogutSusskind fermions in the flavour basis is constructed and some difficulties in the spin-diagonal basis are pointed out.
\end{abstract}

\footnotetext{
*Work supported in part by the European Community's Human Potential Programme under contract HPRN-CT-2000-00131 Quantum Spacetime
} 


\section{Introduction}

In the investigation of some features of QCD at finite baryonic density [1] we must be able to write the baryon charge operator in the transfer matrix formalism. Therefore we must express the transfer matrix in terms of quarkantiquark creation-annihilation operators. Such a formulation has been constructed by by Lüscher [2] for Wilson fermions, but we have not found in the literature anything similar for Kogut-Susskind fermions. Actually we know only one paper on the subject [3], but in this work the transfer matrix is constructed in the spin-diagonal basis, where it is found necessary to introduce auxiliary fields whose relation to quark-antiquarks is not transparent. Another feature, possibly related to the above, might be a difficulty in the way of the applications we are interested in: the transfer matrix is linear in the creation-annihilation operators of quark-antiquarks, rather than exponential, so that the hamiltonian contains the logarithm of these operators.

We have therefore tackled the problem in the flavour basis, and we have extended the formalism developed by Lüscher for Wilson fermions to the case of Kogut-Susskind under the condition that the elementary gauge variables be attached to the links of the blocks rather than of the lattice. Let us explain the reason of this restriction. In the Conclusion we will point out its consequence.

Lüscher prooved the reflection positivity [2] of the transfer matrix in the gauge $U_{0}=\mathbb{1}$. This gauge fixing is too strong, but Menotti and Pelissetto [4] have extended the proof to the gauge $U_{0} \sim \mathbb{1}$, where $U_{0}=\mathbb{1}$ with the exception of a single time slice. If for Kogut-Susskind fermions we define the gauge fields on the links of the blocks, nothing changes in the Menotti-Pelissetto' proof which we have used in our construction. But if we associate the gauge fields to the links of the lattice, their gauge fixing becomes $U_{0}\left(n_{0}\right) U_{0}\left(n_{0}+1\right) \sim \mathbb{1}$ (in standard notation which is however spelled out below). This has a nontrivial Faddeev-Popov determinant which has not been evaluated but must be taken into account to establish the reflection positivity of the transfer matrix.

In this paper we will ignore the pure gauge field part of the action, the partition function and the transfer matrix. Its role in fact is the same as with Wilson fermions, and it can be included in the same way as in Lüscher's paper. 


\section{The transfer matrix}

The quark field $q$ in the flavour basis is defined on hypecubic blocks of coordinates $n_{\mu}$ defined in terms of the sites coordinates $x_{\mu}$

$$
x_{\mu}=2 n_{\mu}+\eta_{\mu}, \quad x_{\mu}=0, \ldots, 2 N_{\mu}-1, \quad n_{\mu}=0, \ldots, N_{\mu}-1, \quad \eta_{\mu}=0,1 .
$$

It carries Dirac indices $\alpha$, flavour indices $f$ and color indices $c$, sometimes comprehensively denoted in the sequel by $I$.

In the transfer matrix formalism most often one has to do with quantities at a given (euclidean) time $n_{0}$. For this reason we use a summation convention over spatial coordinates $\mathbf{n}_{j}$ and intrinsic indices $I$ at fixed time. So for instance we will write

$$
\bar{q}\left(m_{0}\right) Q\left(m_{0}, n_{0}\right) q\left(n_{0}\right)=\sum_{\mathbf{m}, \mathbf{n}, I, J} \bar{q}_{\mathbf{m}, I}\left(m_{0}\right) Q_{\mathbf{m}, I ; \mathbf{n}, J}\left(m_{0}, n_{0}\right) q_{\mathbf{n}, J}\left(n_{0}\right),
$$

where $Q$ is the quark matrix. It is a function of time dependent link operators $U_{\mu}\left(n_{0}\right)$ which have the standard Wilson variables $U_{\mu}\left(m_{0}, \mathbf{m}\right)$ as spatial matrix elements

$$
\left(U_{\mu}\left(m_{0}\right)\right)_{\mathbf{m}, \mathbf{n}}=\delta_{\mathbf{m}, \mathbf{n}} U_{\mu}\left(m_{0}, \mathbf{m}\right) .
$$

In this notation the quark action can be written

$$
S_{q}=\sum_{m_{0}, n_{0}} \bar{q}\left(m_{0}\right) Q\left(m_{0}, n_{0}\right) q\left(n_{0}\right)
$$

Its explicit expression [5] is

$$
S_{q}=\bar{q}\left\{M \mathbb{1} \otimes \mathbb{1}+2 K\left[\gamma_{\mu} \otimes \mathbb{1} \nabla_{\mu}-\gamma_{5} \otimes t_{5} t_{\mu} \delta_{\mu}\right]\right\} q,
$$

where the lattice spacing is equal to $1, M$ and $K$ are the mass and the hopping parameters and $\nabla_{\mu}$ and $\delta_{\mu}$ are the derivatives

$$
\begin{aligned}
\left(\nabla_{\mu} f\right)(n) & =\frac{1}{4}(f(n+2 \mu)-f(n-2 \mu)) \\
\left(\delta_{\mu} f\right)(n) & =\frac{1}{4}(f(n+2 \mu)+f(n-2 \mu)-2 f(n)) .
\end{aligned}
$$

In the tensor product the $\gamma$-matrices act on Dirac indices, while the $t$-matrices act on flavor indices and are given by

$$
t_{\mu}=\gamma_{\mu}^{T}
$$


The minimal gauge interaction (that where the link variables join the quark fields along the shortest path) can be introduced in the standard way by means of the block link variables $U_{\mu}$.

Now we want to identify the components of the quark field which can be related to quarks and antiquarks. They propagate respectively forwards and bakwards in time and are interchanged by charge conjugation. For this purpose we define the projection operators

$$
P_{\mu}^{( \pm)}=\frac{1}{2}\left[\mathbb{1} \otimes \mathbb{1} \pm \gamma_{\mu} \gamma_{5} \otimes t_{5} t_{\mu}\right]
$$

We can thus rewrite the quark matrix singling out the terms which are not diagonal in time in the form

$$
\begin{aligned}
Q\left(m_{0}, n_{0}\right)= & K\left[\gamma_{0} P_{0}^{(-)} U_{0}\left(m_{0}\right) \delta_{n_{0}, m_{0}+1}-\gamma_{0} P_{0}^{(+)} U_{0}^{(+)}\left(n_{0}\right) \delta_{n_{0}, m_{0}-1}\right] \\
& +N\left(n_{0}\right) \delta_{m_{0}, n_{0}},
\end{aligned}
$$

where

$$
\begin{aligned}
N\left(n_{0}\right)= & M \mathbb{1} \otimes \mathbb{1}+K \gamma_{5} \otimes t_{5} t_{0}+K \sum_{j=1}^{3}\left\{\gamma_{5} \otimes t_{5} t_{j}\right. \\
& \left.+\gamma_{j}\left[P_{j}^{(-)} U_{j}\left(n_{0}\right) T_{j}^{(+)}-P_{j}^{(+)} T_{j}^{(-)} U_{j}^{+}\left(n_{0}\right)\right]\right\} .
\end{aligned}
$$

The translation operators $T_{j}^{( \pm)}$have matrix elements

$$
\left(T_{j}^{( \pm)}\right)_{\mathbf{m}, \mathbf{n}}=\delta_{\mathbf{n}, \mathbf{m} \pm e_{j}}
$$

with

$$
\left(e_{j}\right)_{k}=\delta_{j, k}
$$

We see that the components

$$
q^{( \pm)}=P_{0}^{( \pm)} q
$$

of the quark field propagate backwards/forwards respectively. It remains to check that they are properly related by charge conjugation. A possible definition of this operator, with Lüscher's convention for the $\gamma$-matrices is

$$
\mathcal{C}=\gamma_{0} \gamma_{2} \otimes t_{0} t_{2}
$$


whose action on the quark field is

$$
\begin{aligned}
q & =\mathcal{C}^{-1} \bar{q}^{\prime} \\
\bar{q} & =-\mathcal{C}^{T} q^{\prime}
\end{aligned}
$$

Therefore charge conjugation

$$
q^{(+)}=\gamma_{2} \otimes t_{0} t_{2}\left(q^{(-)}\right)^{\prime}
$$

amounts to interchange $q^{( \pm)}$with one another and to rotate the flavours. This action is accettable insofar as the flavour is physically unobservable.

Finally following the Lüscher's procedure we define the transfer matrix $\hat{\mathcal{T}}_{q}$ in terms of creation and annihilation operators related to $q^{( \pm)}$and show that

$$
Z_{q}=\operatorname{Tr} \hat{\mathcal{T}_{q}}=\int[d \bar{q} d q] \exp \left(-16 S_{q}\right)
$$

The factor of 16 in front of $S_{q}$ is the volume of the block. We construct $\hat{\mathcal{T}}_{q}$ in terms of the auxiliary operator

$$
\hat{T}_{q}\left(n_{0}\right)=\exp \left(\hat{y} N\left(n_{0}\right) \hat{x}\right)
$$

according to the ordered product

$$
\hat{\mathcal{T}}_{q}=\mathcal{J} \prod_{n_{0}=0}^{N_{0}-1}\left(\hat{T}_{q}\left(n_{0}\right)\right)^{+} \hat{T}_{q}\left(n_{0}-1\right) .
$$

In the above equations $\mathcal{J}$ is the jacobian of a transformation which will be defined later, and the operator $\hat{T}_{q}\left(n_{0}\right)$ depends on the time $n_{0}$ only throu the dependence on it of the gauge fields. Because $N$ is hermitean, $\hat{\mathcal{T}}_{q}$ is positive definite. The above expression of $\hat{T}_{q}\left(n_{0}\right)$ is valid in the gauge $U_{0}=\mathbb{1}$, assumed by Lüscher to prove reflection positivity. As already said this gauge fixing is too strong, but to lighten the formalism we will nevertheless put $U_{0}=\mathbb{1}$ and we will reinstate $U_{0}$ at the end. The reader can check that keeping $U_{0}$ in the intermediate steps one arrives at the same result.

To prove Eq. (17) we must transform the trace into a Berezin integral. To this end we introduce between the factors in Eq. (19) the identity

$$
\mathbb{1}=\int\left[d x^{+} d x d y^{+} d y\right] \exp \left(-x^{+} x-y^{+} y\right)|x y><x y|,
$$


where the basis vectors

$$
|x y>=| \exp \left(-x \hat{x}^{+}-y \hat{y}^{+}\right)>
$$

are coherent states and the $x, y$ are Grassmann variables. We then use the following equations. First

$$
<x \mid x^{\prime}>=\exp \left(x^{+} x^{\prime}\right) .
$$

Second, if $B=B\left(\hat{x}^{+}\right)$and $C=C(\hat{x})$ are operators which depend on $\hat{x}^{+}, \hat{x}$ only

$$
<x|B \cdot C| x^{\prime}>=B\left(x^{+}\right)<x \mid x^{\prime}>C\left(x^{\prime}\right) .
$$

By means of these equations we can evaluate the kernel of the transfer matrix

$$
\begin{aligned}
< & x\left(n_{0}\right), y\left(n_{0}\right)\left|\hat{\mathcal{T}}_{q}\left(n_{0}\right)\right| x\left(n_{0}-1\right), y\left(n_{0}-1\right)>=\mathcal{J}^{-1} \exp \left(x^{+}\left(n_{0}\right) N\left(n_{0}\right) y^{+}\left(n_{0}\right)\right) \\
& \cdot \exp \left(x^{+}\left(n_{0}\right) x\left(n_{0}-1\right)+\left(y^{+}\left(n_{0}\right) y\left(n_{0}-1\right)\right)\right. \\
& \cdot \exp \left(y\left(n_{0}-1\right) N\left(n_{0}-1\right) x\left(n_{0}-1\right)\right) .
\end{aligned}
$$

Collecting all the pieces we arrive at the euclidean path integral form of the partition function

$$
Z_{q}=\mathcal{J}^{-1} \int\left[d x^{+} d x d y^{+} d y\right] \exp S^{\prime}
$$

with the action

$$
\begin{aligned}
S^{\prime}= & \sum_{n_{0}}-x^{+}\left(n_{0}\right) x\left(n_{0}-1\right)-y^{+}\left(n_{0}\right) y\left(n_{0}-1\right)+x^{+}\left(n_{0}\right) x\left(n_{0}\right)+y^{+}\left(n_{0}\right) y\left(n_{0}\right) \\
& -x^{+}\left(n_{0}\right) N\left(n_{0}\right) y^{+}\left(n_{0}\right)-y\left(n_{0}-1\right) N\left(n_{0}-1\right) x\left(n_{0}-1\right) .
\end{aligned}
$$

The change of variables

$$
x=4 \sqrt{K} q^{(+)}, y^{+}=4 \sqrt{K} q^{(-)},
$$

whose jacobian is the function $\mathcal{J}$ introduced in Eq.(19) transforms $S^{\prime}$ into $S_{q}$ thus proving Eq.(17). 


\section{Conclusion}

We have extended the Lusher's formalism for Wilson fermions to the case of Kogut-Susskind in the flavour basis under the condition that the gauge fields be associated to the links of the blocks. This restriction is due to the use of the Menotti-Pelissetto proof of reflection positivity which we used in the construction.

This result can be sufficient for our purposes [1] but leaves unsolved the case of the spin-diagonal basis. In fact we cannot get the spin-diagonal formulation by a simple change of basis [5]. There is a formal difficulty and a practical complication in the way. To this end one should associate the gauge fields to the links of the lattice and this requires an extension of the proof of reflection positivity in the presence of the Faddeev-Popov determinant in the gauge $U_{0}\left(n_{0}\right) U_{0}\left(n_{0}+1\right) \sim \mathbb{1}$. Assuming this to be done, however, we would get an awkward result because a minimal gauge coupling in one basis becomes cumbersome in the other [6]. One should then start from the minimal gauge coupling in the spin-diagonal basis, find the coupling resulting in the flavour basis, and show reflection positivity with such coupling. Both shortcomings are hopefully surmountable, but the problem requires further study and we think that deserves a wider interest than our specific motivation.

The alternative is to construct the transfer matrix directly in the spindiagonal basis in a form convenient for the use at finite baryon density. 


\section{References}

[1] F.Palumbo, hep-lat/0202021; hep-lat/0208002

[2] M.Lüscher, Commun. Math. Phys. 54 (1977) 283

[3] H.S.Sharatchandra, H.T.Thun and P.Weisz, Nucl.Phys.B192(1981)205

[4] P.Menotti and A.Pelissetto, Commun. Math. Phys.113(1987)369

[5] I.Montvay and G.Münster, Quantum fields on a lattice, Cambridge University press, 1994

[6] H.Kluberg-Stern, A.Morel, O.Napoly and B.Petersson, Nucl. Phys. B220[FS8] (1983) 447; G.T.Bodwin and E.V.Kovacs, Phys. Rev. 38D (1988) 1206 